\title{
Reversed sex-biased mutation rates for indels and base substitutions in Drosophila melanogaster
}

Lauri Törmä ${ }^{1,2}$, Claire Burny $^{1,2}$, Christian Schlötterer ${ }^{1, \#}$

1) Institut für Populationsgenetik, Vetmeduni Vienna, Vienna, Austria

2) Vienna Graduate School of Population Genetics, Vetmeduni Vienna, Vienna, Austria

\#) corresponding author.

Keywords: Sex-biased mutations, spellchecker1, faster-X evolution, Drosophila melanogaster

\section{Correspondence:}

Christian Schlötterer

Institut für Populationsgenetik, Vetmeduni Vienna, 1210 Wien, Austria

Email: christian.schloetterer@vetmeduni.ac.at 


\section{Abstract}

2 Sex biases in mutation rates may affect the rate of adaptive evolution. In many species, males

3 have higher mutation rates than females when single nucleotide variants (SNVs) are considered.

4 In contrast, indel mutations in humans and chimpanzees are female-biased. In Drosophila

5 melanogaster, direct estimates of mutation rates did not uncover sex differences, but a recent

6 analysis suggested the presence of male-biased SNVs mutations. Here we study the sex-specific

7 mutation processes using mutation accumulation data from mismatch-repair deficient $D$.

8 melanogaster. We find that sex differences in flies are similar to the ones observed in humans: a

9 higher mutation rate for SNVs in males and a higher indel rate in females. These results have

10 major implications for the study of neutral variation and adaptation in Drosophila. 


\section{Introduction}

12

13

14

15

16

17

Mutations are the ultimate source of novelty in evolution. However, mutations do not occur at a constant rate across the genome $(1,2)$, between taxa (3), or sexes (4). The neutral substitution rate is determined by the primary mutation rate (5) and it needs to be considered when studying the intensity of selection from divergence data. If mutation rates differ between sexes, genes are expected to evolve at different rates depending on which chromosome they are located (6). For example, a higher mutation rate in males would result in a lower substitution rate on the $\mathrm{X}$ chromosome compared to the autosomes, while the $\mathrm{Y}$ chromosome has the highest substitution rate.

Higher male mutation rates have been observed for SNVs in mammals (7-9), birds (10, 11), salmonid fishes $(12)$ and plants $(13,14)$. For indels, the observed sex-biases show no consistent trend across species. Human and chimpanzee females have a higher indel rate than males (1517). In barn swallows, a sex-specific effect was shown for the mutation rate of a single microsatellite locus $(18,19)$ while in mice and rats indels are male-biased $(20)$.

While sex-specific mutation rates are frequently observed, the underlying processes are highly taxon and mutation-type specific. In mammals, the higher rate of SNV mutations in males has been explained by more germline cell divisions in testes $(9,21)$. This explanation was challenged by the observation that even in young parents which have a similar number of germline cell divisions, males have a three times higher mutation rate than females (22). Moreover, the maleto-female mutation rate ratio barely increases with age, which is in conflict with the explanation involving germline divisions (22). In plants, explanations for sex-specific mutation rates include differences in per-replication mutation rates between the sex chromosomes (13), as well as different contributions of somatic mutations in males and females (14). For indels, the quiescent state of the oocyte may be responsible for the higher female mutation rate in humans (17). Similar 
to yeast (23), the quiescent oocyte is expected to acquire mutations not during replication, but from other sources, such as double-stranded breaks (17). In mice and rats, the male-bias of indel mutation rates is consistent with the hypothesis that DNA replication errors are the major source of mutations (20).

Consistent with a similar number of cell divisions for male and female germlines - on average 35.5

43 in males and 34.5 in females (24) - early sequencing studies in Drosophila found similar mutation

44 rates for both sexes (25-29). Whole-genome comparisons with other Drosophila species provided

45 conflicting results. These studies reported higher (30), equal (31), and lower (32) neutral substitution rates for the $\mathrm{X}$ chromosome. A recent analysis comparing $D$. melanogaster, $D$. simulans, and $D$. yakuba genomes challenged some of these findings and suggested that the $\mathrm{X}$ to autosomal (X/A) ratio of mutation rates could range from 0.81 to 0.93 , depending on the type of neutral site used for the estimation (33). Further evidence for male-biased base substitution rates comes from the comparison of neo-Y and neo-X chromosomes in D. miranda, which showed higher substitution rates on the neo-Y chromosome (34). Since polymorphism and divergence patterns are strongly affected by selection, it is particularly noteworthy that a trend for male-biased

In this study, we estimate de novo mutation rates on the $\mathrm{X}$ chromosome and the autosomes using data from a mutation accumulation study mismatch-repair (MMR) deficient $D$. melanogaster strain consisting of 7,345 new single nucleotide variants (SNV) and 5,672 indels (36). We find opposing patterns of sex-specific mutation rates for SNVs and indels. While males have a higher mutation rate for SNVs (X/A mutation rates ratio: 0.876), indels are more common in females (X/A mutation rates ratio: 1.959). We show that wild-type females also have a higher indel mutation rate and

61 conclude that our results are not an artifact from the mismatch repair deficiency, but reflect 62 genuine sex-specific differences in mutation processes. These results have major implications for 
63 the interpretation of neutral variation and adaptation in $D$. melanogaster.

\section{Results and discussion}

66 We scrutinized sex-specific mutation rates in $D$. melanogaster by comparing the de novo

67 mutations on the $\mathrm{X}$ chromosome and the autosomes in a mismatch repair deficient genetic

68 background, lacking a functional copy of the MutS homolog spellchecker1 (37). Because the X

69 chromosome spends more time in females than in males, a higher mutation rate in males leads

70 to a higher mutation rate on the autosomes compared to the $\mathrm{X}$ chromosome. Consistent with

71 previous results (33), we found that the X/A ratio of mutation rates was 0.876 (Poisson test, 95\%

72 Confidence Interval $(\mathrm{Cl})$ : $0.823-0.933, \mathrm{p}$-value $=2.923 \times 10^{-5}$ ) resulting from a $\mathrm{X}$ chromosomal

73 mutation rate of $8.71 \times 10^{-7}\left(95 \%\right.$ Poisson $\left.\mathrm{Cl}: 8.23 \times 10^{-7}-9.23 \times 10^{-7}\right)$ and an autosomal rate of

$749.94 \times 10^{-7}\left(95 \%\right.$ Poisson Cl: $\left.9.69 \times 10^{-7}-1.02 \times 10^{-6}\right)$ (Figure 1a \& Table 1). The difference between

75 chromosomes still persists after controlling for the base content between the chromosomes

76 (Figure 1b \& Table 1).

78 With indel rates being sex-specific in multiple species (17-20), we tested for sex-specific indel

79 rates in Drosophila. The indel mutation rate on the $\mathrm{X}$ chromosome was $1.26 \times 10^{-6}(95 \%$ Poisson

$\left.80 \mathrm{Cl}: 1.20 \times 10^{-6}-1.32 \times 10^{-6}\right)$, which is almost twice as high as the autosomal rate of $6.42 \times 10^{-7}(95 \%$

81 Poisson $\left.\mathrm{Cl}: 6.22 \times 10^{-7}-6.62 \times 10^{-7}\right)$, resulting in a significant rate ratio of $1.959(95 \%$ Poisson $\mathrm{Cl}$ :

82 1.850-2.074, p-value $\left.<2.2 \times 10^{-16}\right)($ Figure 1c \& Table 1).

84 Indels occur more frequently on stretches of short tandem repeats (microsatellites) due to DNA 85 replication slippage (38-40). Because microsatellite repeat number is an important factor in 86 determining the indel mutation rate (41-43), the inferred sex-specific indel mutation rate may be 87 the result of the heterogeneity in repeat length distribution between the $\mathrm{X}$ chromosome and the 88 autosomes (44). We accounted for possible differences in the distribution of repeat length on the 
$\mathrm{X}$ and the autosomes by fitting a binomial generalized additive model (GAM, (45); see Methods) separately for mono- and di-nucleotide repeats. The indel mutation rate changes significantly with repeat length for both the homopolymers and dinucleotides ( $>5$ degrees of freedom (dof), $x 2=$ $5,046, p<2 \times 10^{-16} ;>6$ dof, $x 2=1,676, p<2 \times 10^{-16}$ respectively), but not in a monotonous way. The mutation rate increases for homopolymer runs up to 10-12 bp for (Figure 2a) and for dinucleotides up to 9-10 repeat units (Figure $2 b$ ). Microsatellites with more repeats were less likely to mutate and also less common. We found that the indel rate is significantly higher on the $\mathrm{X}$ chromosome for homopolymers (Odds Ratio X/A $(\mathrm{OR})=1.163,95 \% \mathrm{Cl}$ : 1.093-1.237, deviance analysis

$\left.97 \mathrm{p}=2.049 \times 10^{-6}\right)($ Figure $2 \mathrm{a})$ and dinucleotides $(\mathrm{OR}=1.291,95 \% \mathrm{Cl}: 1.074-1.553$, deviance analysis

$\left.98 p=7.184 \times 10^{-3}\right)$ (Figure $2 b$ ). The highly consistent pattern across the two different repeat types strongly suggests that female flies have a higher indel mutation rate than males.

100

To rule out that the sex-specific difference in indel rates is an artifact of the mismatch repair system deficiency, we studied indel mutations in a natural Drosophila population. Using variant number of indels to the number of SNVs between the $\mathrm{X}$ chromosome and the autosomes. We found an odds ratio significantly different from $1(\mathrm{OR}=1.320,95 \% \mathrm{Cl}: 1.309-1.332$, Fisher's exact test, $\left.p<2.2 \times 10^{-16}\right)$, indicating that more indels occur on the $X$ chromosome which confirms the female-biased indel mutation rate also in wild-type flies.

While we find the same sex-biased indel mutation rate as in humans and chimpanzees (17), we consider it unlikely that the interpretation of this sex-specific difference can be applied to

111 Drosophila. For humans and chimpanzees, the quiescent state of the female oocyte has been 112 attributed to the higher indel rate in females (17). This is however unlikely to cause the 113 pronounced differences between the $\mathrm{X}$ chromosome and the autosomes in our experiment 114 because it was designed such that young females were fertilized, leaving little opportunity for 
oocytes to enter a quiescent state. The mismatch repair targets replicating cells (47), which implies that most of the observed mutations did not occur in the quiescent phase, but during

117 replication. Furthermore, the majority of indels (95.47\% in mono- and di-nucleotides) occur on

118 microsatellites arguing in favor of replication errors. Hence, we conclude that, at least in

119 Drosophila, the higher indel rate in females cannot be explained by the quiescent phase of the 120 oocytes, but rather other sex-specific differences in replication and/or DNA repair must be 121 responsible for these differences.

123 One particularly interesting aspect of our study is that we detect sex-specific mutation rates for two types of mutations, SNVs and indels, but the sex-bias is reversed between them (Figures 1a, 1c). It has been proposed that the mutation rate has evolved as a balance between the cost in fitness due to accurate replication, repair, and deleterious mutations and the benefit of fitness increase due to advantageous mutations (48). In the case of sex-biased mutation rates, this implies that the cost/benefit balance differs between sexes (49). Since we show a different sex bias for SNVs and indels, it is difficult to reconcile a simple cost/benefit balance with our data. Rather, we consider it more likely that DNA damage and repair are sex-specific processes with their own signatures. The same conclusion was reached recently in humans (22).

133 The impact of the different sex bias for SNVs and indels is nicely illustrated by the different length 134 distributions of AT-microsatellites on the X chromosome and autosomes in D. melanogaster (44). 135 Microsatellite length may be explained by an equilibrium process between replication slippage 136 generating longer repeats and base substitutions shortening the microsatellite (50). Hence, it was 137 previously proposed that the difference in length distribution in $D$. melanogaster may be either 138 explained by a higher slippage rate on the $X$ chromosome or a higher base substitution rate in 139 males (44). Our study now demonstrates that both processes occur and their joint effects probably 
140

141

142

143 In this study, we demonstrated that increasing the mutation rate in mutation accumulation lines is

144 a powerful approach to study mutation processes, as it overcomes the typically encountered

145 limitation of too few observations. We anticipate that the analysis of mutation accumulation lines

146 with elevated mutation rates can provide a powerful method to study differences in the mutation

147 process, either between sexes, as in this study, or between different genomic regions.

148

149

150

151

152

153

154

155

156

157

158

159

160

161

162

\section{Microsatellites and the model}

164 We used a previously published $R$ code (43) to search for homopolymer runs from 165 BSgenome.Dmelanogaster.UCSC.dm6 (54). Dinucleotide repeats were annotated from the $D$. 

of 18 repeats (homopolymers) or 17 repeat units (dinucleotides), as longer microsatellites did not have any indel mutations. Following (43) we analyzed the occurrence of 5,415 $(95.47 \%)$ indels separately for homopolymers and dinucleotides using binomial generalized additive models (45) with the non-linear effect of the repeat length. In addition we tested for differences between

172 chromosomes by using the chromosomal status (autosomes or $\mathrm{X}$ ) as covariate. The 173 corresponding R function is mgcv::gam(cbind(\#indels, \#repeats - \#indels) Chr + s(Length, fx = 174 FALSE, $\mathrm{k}=-1$, bs = "cr"), family = binomial, data) $(45,56)$ (mgcv R package version 1.8-31), where $175 s$ is a cubic spline, "\#indels" is the number of indels per number of repeats, "\#repeats" is the number of repeats of a given length and "Chr", the chromosomal status. Two models, with (M1) and without (M0) the chromosome covariate "Chr", were compared in a deviance analysis using the mgcv::anova.gam(M0, M1, test = "Chisq") R function. Odds ratios (OR) and their $95 \%$ indels in both types of repeats normalized to the genome-wide number of repeats (Figure 2, yaxis).

\section{Code and data availability}

187 The code ( $R$ and bash scripts) and intermediate files such as indel counts per repeat type will be 188 accessible in the following github repository: ${ }^{* * *}$, available upon publication.

\section{Author contribution}

191 L. T., C. B. analyzed the data, L. T., C. B., C. S. wrote the paper, C.S. supervised the project. 


\section{Acknowledgments}

193 This work was supported by the Austrian Science Fund (FWF, grant W1225). We thank A.

194 Futschik for statistical advice.

195

\section{References}

1. M. Wierdl, M. Dominska, T. D. Petes, Microsatellite instability in yeast: dependence on the length of the microsatellite. Genetics 146, 769-779 (1997).

2. Z. J. Assaf, S. Tilk, J. Park, M. L. Siegal, D. A. Petrov, Deep sequencing of natural and experimental populations of Drosophila melanogaster reveals biases in the spectrum of new mutations. Genome Res. 27, 1988-2000 (2017).

3. W. Sung, M. S. Ackerman, S. F. Miller, T. G. Doak, M. Lynch, Drift-barrier hypothesis and mutation-rate evolution. Proc. Natl. Acad. Sci. U. S. A. 109, 18488-18492 (2012).

4. L. D. Hurst, H. Ellegren, Sex biases in the mutation rate. Trends Genet. 14, 446-452 (1998).

5. M. Kimura, Evolutionary rate at the molecular level. Nature 217, 624-626 (1968).

6. M. Kirkpatrick, D. W. Hall, Male-biased mutation, sex linkage, and the rate of adaptive evolution. Evolution 58, 437-440 (2004).

7. R. A. Gibbs, et al., Genome sequence of the Brown Norway rat yields insights into mammalian evolution. Nature 428, 493-521 (2004).

8. K. Lindblad-Toh, et al., Genome sequence, comparative analysis and haplotype structure of the domestic dog. Nature 438, 803-819 (2005).

9. M. A. Wilson Sayres, C. Venditti, M. Pagel, K. D. Makova, Do variations in substitution rates and male mutation bias correlate with life-history traits? A study of 32 mammalian genomes. Evolution 65, 2800-2815 (2011).

10. H. Ellegren, A. K. Fridolfsson, Male-driven evolution of DNA sequences in birds. Nat. Genet. 17, 182-184 (1997).

11. N. W. Kahn, T. W. Quinn, Male-driven evolution among Eoaves? A test of the replicative division hypothesis in a heterogametic female (ZW) system. J. Mol. Evol. 49, 750-759 (1999).

12. H. Ellegren, A.-K. Fridolfsson, Sex-specific mutation rates in salmonid fish. J. Mol. Evol. 56, 458-463 (2003).

13. D. A. Filatov, D. Charlesworth, Substitution rates in the $X$ - and $Y$-linked genes of the plants, Silene latifolia and S. dioica. Mol. Biol. Evol. 19, 898-907 (2002).

14. C.-A. Whittle, M. O. Johnston, Male-driven evolution of mitochondrial and chloroplastidial DNA sequences in plants. Mol. Biol. Evol. 19, 938-949 (2002). 
15. T. Grimm, et al., On the origin of deletions and point mutations in Duchenne muscular dystrophy: most deletions arise in oogenesis and most point mutations result from events in spermatogenesis. J. Med. Genet. 31, 183-186 (1994).

16. C. Lázaro, et al., Sex differences in mutational rate and mutational mechanism in the NF1 gene in neurofibromatosis type 1 patients. Hum. Genet. 98, 696-699 (1996).

17. G. Achaz, S. Gangloff, B. Arcangioli, The quiescent $X$, the replicative $Y$ and the Autosomes https:/doi.org/10.1101/351288.

18. C. R. Primmer, N. Saino, A. P. Moller, H. Ellegren, Unraveling the Processes of Microsatellite Evolution Through Analysis of Germ Line Mutations in Barn Swallows Hirundo rustica. Mol. Biol. Evol. 15, 1047-1047 (1998).

19. J. Brohede, C. R. Primmer, A. Møller, H. Ellegren, Heterogeneity in the rate and pattern of germline mutation at individual microsatellite loci. Nucleic Acids Res. 30, 1997-2003 (2002).

20. K. D. Makova, S. Yang, F. Chiaromonte, Insertions and deletions are male biased too: a whole-genome analysis in rodents. Genome Res. 14, 567-573 (2004).

21. J. B. S. Haldane, The rate of spontaneous mutation of a human gene. J. Genet. 31, 317 (1935).

22. Z. Gao, et al., Overlooked roles of DNA damage and maternal age in generating human germline mutations. Proc. Natl. Acad. Sci. U. S. A. 116, 9491-9500 (2019).

23. S. Gangloff, et al., Quiescence unveils a novel mutational force in fission yeast. Elife 6 (2017).

24. J. B. Drost, W. R. Lee, The developmental basis for germline mosaicism in mouse and Drosophila melanogaster. Genetica 102-103, 421-443 (1998).

25. V. L. Bauer, C. F. Aquadro, Rates of DNA sequence evolution are not sex-biased in Drosophila melanogaster and D. simulans. Mol. Biol. Evol. 14, 1252-1257 (1997).

26. D. J. Begun, P. Whitley, Reduced X-linked nucleotide polymorphism in Drosophila simulans. Proc. Natl. Acad. Sci. U. S. A. 97, 5960-5965 (2000).

27. A. J. Betancourt, D. C. Presgraves, W. J. Swanson, A test for faster X evolution in Drosophila. Mol. Biol. Evol. 19, 1816-1819 (2002).

28. B. A. Counterman, D. Ortíz-Barrientos, M. A. F. Noor, Using comparative genomic data to test for fast-X evolution. Evolution 58, 656-660 (2004).

29. K. Thornton, D. Bachtrog, P. Andolfatto, $X$ chromosomes and autosomes evolve at similar rates in Drosophila: no evidence for faster-X protein evolution. Genome Res. 16, 498-504 (2006).

30. D. J. Begun, et al., Population genomics: whole-genome analysis of polymorphism and divergence in Drosophila simulans. PLoS Biol. 5, e310 (2007). 
Drosophila simulans genome provides new insights into patterns of lineage-specific divergence. Genome Res. 23, 89-98 (2013).

32. D. Garrigan, S. B. Kingan, A. J. Geneva, J. P. Vedanayagam, D. C. Presgraves, Genome diversity and divergence in Drosophila mauritiana: multiple signatures of faster $\mathrm{X}$ evolution. Genome Biol. Evol. 6, 2444-2458 (2014).

33. B. Charlesworth, J. L. Campos, B. C. Jackson, Faster-X evolution: Theory and evidence

34. D. Bachtrog, Evidence for male-driven evolution in Drosophila. Mol. Biol. Evol. 25, 617-619 (2008).

35. P. D. Keightley, et al., Analysis of the genome sequences of three Drosophila melanogaster spontaneous mutation accumulation lines. Genome Res. 19, 1195-1201 (2009).

36. L. Törmä, C. Burny, V. Nolte, K.-A. Senti, C. Schlötterer, Transcription-coupled repair in Drosophila melanogaster is independent of the mismatch repair pathway. bioRxiv, 2020.04.07.029033 (2020).

37. C. Flores, W. Engels, Microsatellite instability in Drosophila spellchecker1 (MutS homolog) mutants. Proc. Natl. Acad. Sci. U. S. A. 96, 2964-2969 (1999).

38. G. Levinson, G. A. Gutman, Slipped-strand mispairing: a major mechanism for DNA sequence evolution. Mol. Biol. Evol. 4, 203-221 (1987).

39. C. Schlötterer, Evolutionary dynamics of microsatellite DNA. Chromosoma 109, 365-371

40. H. Ellegren, Microsatellites: simple sequences with complex evolution. Nat. Rev. Genet. 5, 435-445 (2004).

41. B. Brinkmann, M. Klintschar, F. Neuhuber, J. Hühne, B. Rolf, Mutation rate in human microsatellites: influence of the structure and length of the tandem repeat. Am. J. Hum. Genet. 62, 1408-1415 (1998).

42. B. Harr, C. Schlötterer, Long microsatellite alleles in Drosophila melanogaster have a downward mutation bias and short persistence times, which cause their genome-wide underrepresentation. Genetics 155, 1213-1220 (2000).

43. B. Meier, et al., Mutational signatures of DNA mismatch repair deficiency in C. elegans and human cancers. Genome Res. 28, 666-675 (2018).

44. D. Bachtrog, S. Weiss, B. Zangerl, G. Brem, C. Schlötterer, Distribution of dinucleotide microsatellites in the Drosophila melanogaster genome. Mol. Biol. Evol. 16, 602-610 (1999).

45. S. N. Wood, Fast stable restricted maximum likelihood and marginal likelihood estimation of semiparametric generalized linear models. J. R. Stat. Soc. Series B Stat. Methodol. 73, 336 (2011). 482, 173-178 (2012). 
47. P. Modrich, Mechanisms in eukaryotic mismatch repair. J. Biol. Chem. 281, 30305-30309 (2006).

48. P. D. Sniegowski, P. J. Gerrish, T. Johnson, A. Shaver, The evolution of mutation rates: separating causes from consequences. Bioessays 22, 1057-1066 (2000).

49. H. Ellegren, Characteristics, causes and evolutionary consequences of male-biased mutation. Proc. Biol. Sci. 274, 1-10 (2007).

50. S. Kruglyak, R. T. Durrett, M. D. Schug, C. F. Aquadro, Equilibrium distributions of microsatellite repeat length resulting from a balance between slippage events and point mutations. Proc. Natl. Acad. Sci. U. S. A. 95, 10774-10778 (1998).

51. R Core Team, R: A Language and Environment for Statistical Computing (2018).

52. A. McKenna, et al., The Genome Analysis Toolkit: a MapReduce framework for analyzing next-generation DNA sequencing data. Genome Res. 20, 1297-1303 (2010).

53. H. Long, M. G. Behringer, E. Williams, R. Te, M. Lynch, Similar Mutation Rates but Highly Diverse Mutation Spectra in Ascomycete and Basidiomycete Yeasts. Genome Biol. Evol. 8, 3815-3821 (2016).

54. The Bioconductor Dev Team, BSgenome.Dmelanogaster.UCSC.dm6: Full genome sequences for Drosophila melanogaster (UCSC version dm6) (2014).

55. T. Thiel, W. Michalek, R. K. Varshney, A. Graner, Exploiting EST databases for the development and characterization of gene-derived SSR-markers in barley (Hordeum vulgare L.). Theor. Appl. Genet. 106, 411-422 (2003).

56. S. N. Wood, N. Pya, B. Säfken, Smoothing Parameter and Model Selection for General Smooth Models. J. Am. Stat. Assoc. 111, 1548-1563 (2016).

57. O. Miettinen, M. Nurminen, Comparative analysis of two rates. Stat. Med. 4, 213-226 (1985). 
a

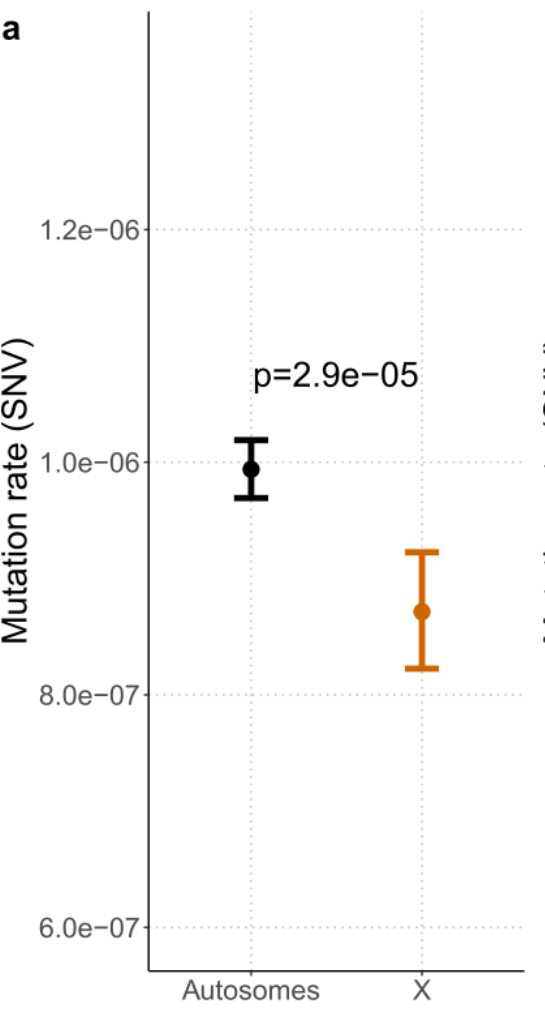

b

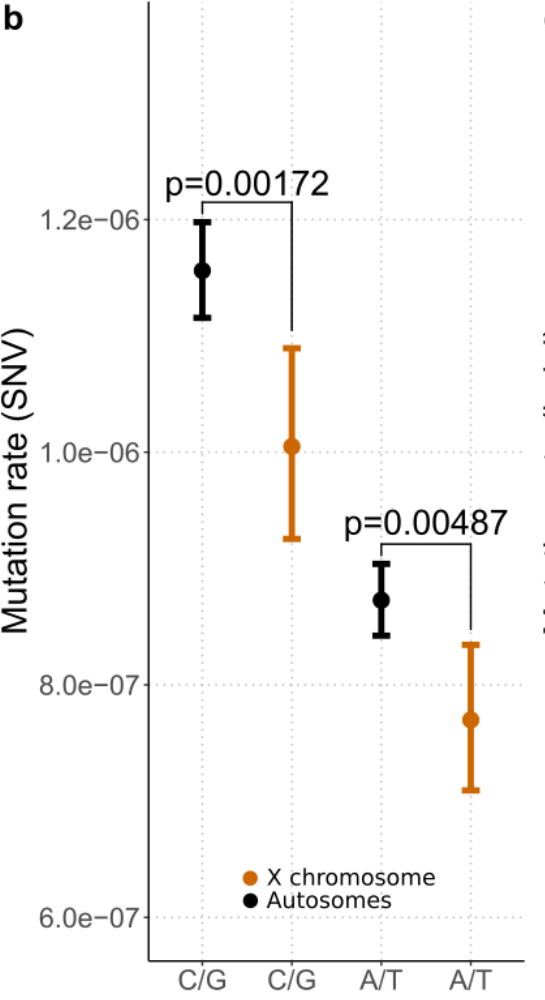

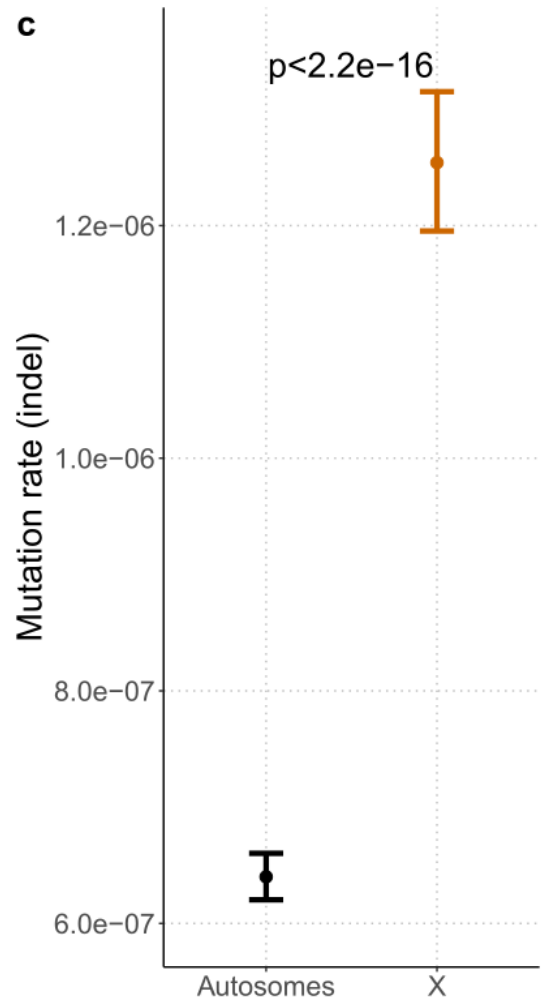

327 Figure 1. Mutation rate differences between the autosomes (black) and the $\mathrm{X}$ chromosome 328 (orange). a) SNV mutation rates across all sites and b) separated for A/T and G/C pairs. c) Indel 329 mutation rates across all sites. The bars represent $95 \%$ confidence intervals using exact Poisson tests, the dots represent the actual estimates. 
a

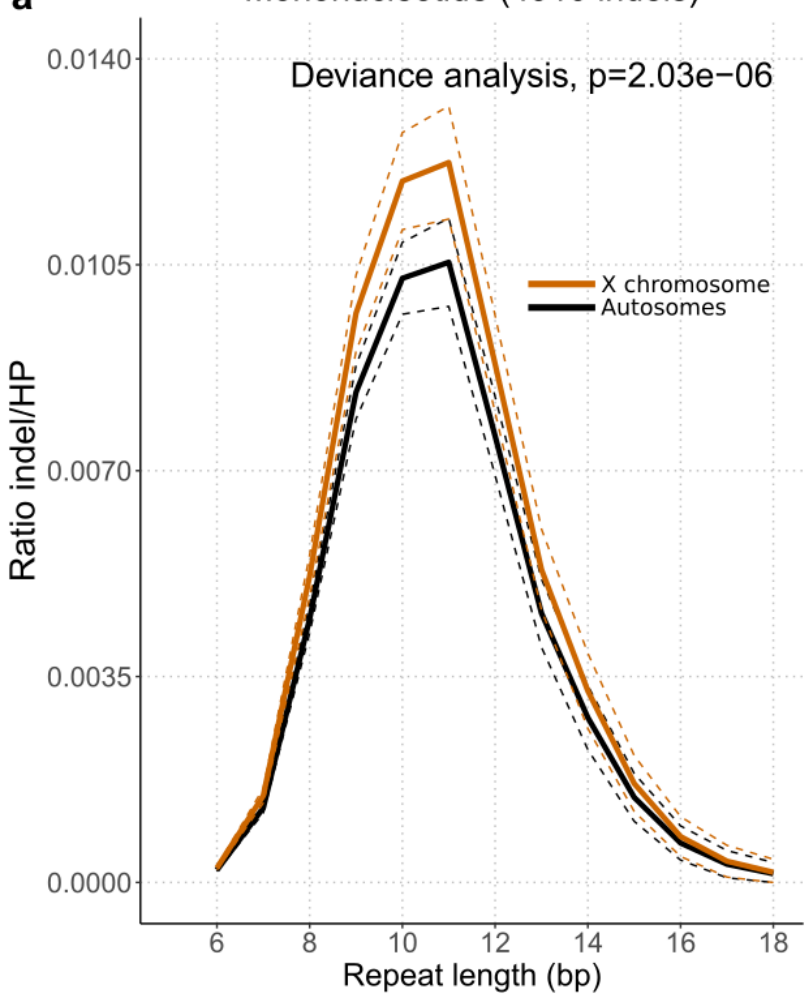

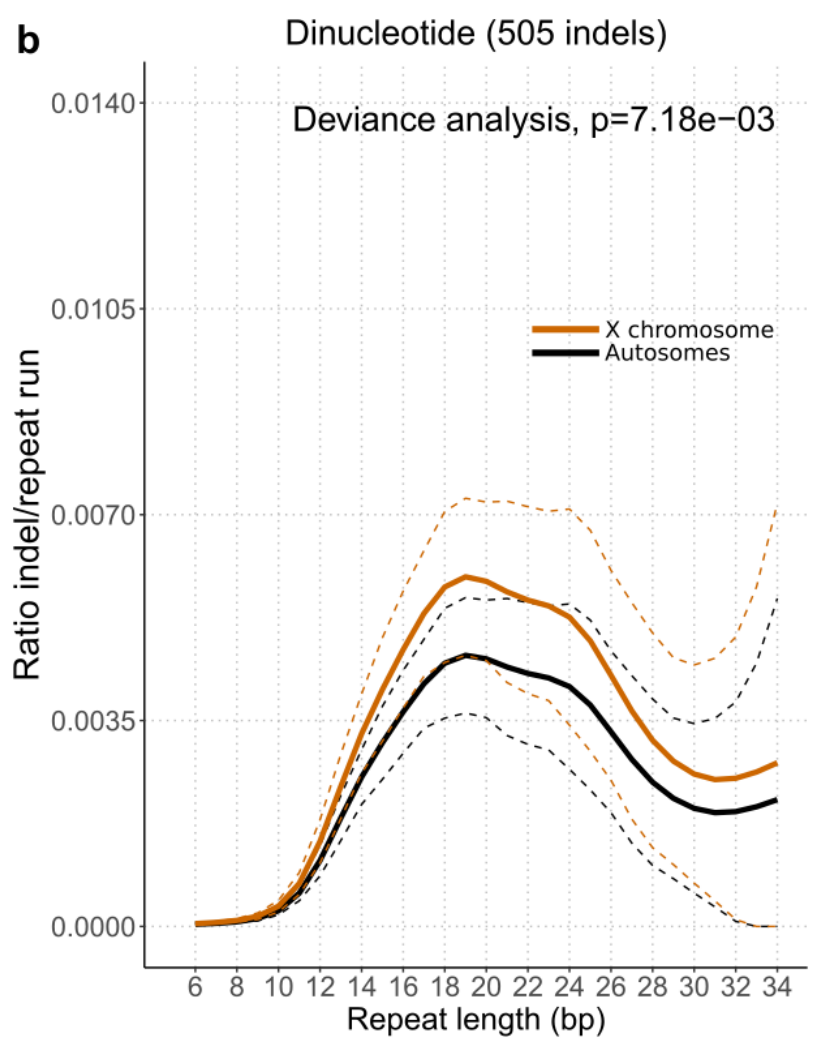

332 Figure 2. Occurence of indels in mononucleotide (a) and dinucleotide (b) repeats on the 333 autosomes (black) and the $\mathrm{X}$ chromosome (orange). The $\mathrm{x}$-axis represents the repeat length. The $334 y$-axis represents the ratio of indels normalized for the prevalence of repeat type in the genome, 335 as described in (43). Binomial generalized additive models with cubic spline have been fitted. The 336 predicted values are in plain lines, 95\% confidence intervals are represented in dotted lines. The 337 reported $p$-values are obtained from a deviance analysis contrasting a model with and without the 338 chromosomal status (autosomes or X) covariate. 
Table 1. Mutation rate estimates.

\begin{tabular}{|l|l|c|l|}
\hline $\begin{array}{l}\text { Mutation } \\
\text { type }\end{array}$ & Context & Number of mutations & Mutation rate $(95 \%$ Poisson CI) \\
\hline SNVs & Autosomes & 6161 & $9.938 \times 10^{-7}\left(9.691 \times 10^{-7}-1.019 \times 10^{-6}\right)$ \\
\hline SNVs & X chromosome & 1184 & $8.714 \times 10^{-7}\left(8.225 \times 10^{-7}-9.225 \times 10^{-7}\right)$ \\
\hline SNVs & C/G Autosomes & 3063 & $1.156 \times 10^{-6}\left(1.116 \times 10^{-6}-1.198 \times 10^{-6}\right)$ \\
\hline SNVs & C/G X chromosome & 590 & $1.005 \times 10^{-6}\left(9.255 \times 10^{-7}-1.089 \times 10^{-6}\right)$ \\
\hline SNVs & A/T Autosomes & 3098 & $8.727 \times 10^{-7}\left(8.423 \times 10^{-7}-9.040 \times 10^{-7}\right)$ \\
\hline SNVs & A/T X chromosome & 594 & $7.699 \times 10^{-7}\left(7.092 \times 10^{-7}-8.343 \times 10^{-7}\right)$ \\
\hline Indel & Autosomes & 3968 & $6.401 \times 10^{-7}\left(6.203 \times 10^{-7}-6.603 \times 10^{-7}\right)$ \\
\hline Indel & X chromosome & 1704 & $1.254 \times 10^{-6}\left(1.195 \times 10^{-6}-1.315 \times 10^{-6}\right)$ \\
\hline
\end{tabular}

